\title{
Erosion, formation of deposited layers and fuel retention for beryllium under the influence of plasma impurities
}

\author{
A. Kreter ${ }^{1}$, T. Dittmar ${ }^{1,2}$, D. Nishijima ${ }^{2}$, R.P. Doerner ${ }^{2}$, M.J. Baldwin ${ }^{2}$ and K. Schmid ${ }^{3}$ \\ ${ }^{1}$ Institute for Energy and Climate Research - Plasma Physics, Forschungszentrum Juelich, Association \\ EURATOM-FZJ, Trilateral Euregio Cluster, Germany \\ ${ }^{2}$ Center for Energy Research, University of California at San Diego, 9500 Gilman Drive, La Jolla, CA 92093- \\ 0417, USA \\ ${ }^{3}$ Max-Planck-Institut für Plasmaphysik, EURATOM Association, Garching, Germany \\ E-mail: a.kreter@fz-juelich.de
}

\section{Abstract}

The impact of argon as a seeded plasma impurity on the plasma-material interaction properties of beryllium was studied in the PISCES-B linear plasma device. When exposed to pure deuterium plasma, the surface of beryllium forms a fine-scale grass-like structure and contains a significant fraction of deuterium, leading to the reduction of the sputtering yield. An argon fraction in plasma of $10 \%$ prevents the formation of rough surface and reduces the deuterium retention in the beryllium target by $>50 \%$. Consequently, the beryllium sputtering yield in the presence of argon remains at a level close to the prediction by the binary collision code TRIM. The total retention of deuterium in co-deposited layers remains at a similar level when argon is added, since a higher amount of deposited $\mathrm{Be}$ is compensated by a lower $\mathrm{D} / \mathrm{Be}$ value. Aluminium also forms a fine-scale surface structure and displays a reduction of the effective sputtering yield when exposed to pure deuterium plasma and can therefore be considered as a non-toxic proxy for studying selected plasma-material interaction properties of beryllium.

\section{Introduction}

Beryllium will be used as the plasma-facing material of the main ITER chamber [1]. Beryllium's main advantages are its good compatibility with the fusion plasma due to its low $\mathrm{Z}(\mathrm{Z}=4)$, relatively good thermo-mechanical properties, such as the highest melting temperature of all low-Z metals $(1560 \mathrm{~K})$, and the ability to getter oxygen. Because of the toxicity of beryllium, the experimental database on its plasma-material interaction (PMI) 
properties is, however, fairly limited. An overview on beryllium as plasma-facing material is given in [2].

Values of the sputtering yield of beryllium calculated in the binary collision approximation, e.g. by the TRIM code [3], are often used as the reference when comparing with experimental findings. While measurements of the Be sputtering yield by the hydrogen isotopes in low-flux ion beam facilities are in good agreement with TRIM [2], values measured in the high-flux linear plasma device PISCES-B are persistently by about one order of magnitude lower than the TRIM predictions [4]. This discrepancy was explained by the evolving surface morphology and the protective role of hydrogen incorporated in the Be surface [5]. The increased roughness of fine-scale grass-like surface structures can lead to a higher fraction of promptly re-deposited $\mathrm{Be}$ atoms, reducing the net erosion by a factor of $2-3$. This effect is similar to the enhanced prompt re-deposition found on rough surfaces in ${ }^{13} \mathrm{CH}_{4}$ tracer injection experiments in the TEXTOR tokamak (figure 5 in [6]). The second effect reducing the Be sputtering is the dilution of beryllium by hydrogen in the interaction layer and the less efficient change of the momentum direction of the incident particles necessary for sputtering. Again, a parallel can be drawn to the ${ }^{13} \mathrm{CH}_{4}$ experiments in TEXTOR with an increased sputtering of carbon when deposited on heavy tungsten (figure 12 in [7]). Calculations by the TRIM code predict a reduction of Be sputtering by a factor of 4 for a $50 \%$ concentration of deuterium in the surface [5]. Superposition of the effects of the surface morphology and composition may explain the reduction of Be sputtering yields by about one order of magnitude compared to TRIM. Note that in ion beam experiments both of these effects are less pronounced because of a significantly lower ion flux and fluence to the surface.

Studies of the deuterium retention in the beryllium bulk were also performed in ion beam facilities and linear plasma devices, summarized in [8]. For low incident fluences the retention scales linearly with fluence, with a tendency to saturate at a level of $10^{20}-10^{21} \mathrm{D} / \mathrm{m}^{2}$ for fluences higher than $10^{21}-10^{22} \mathrm{D}^{+} / \mathrm{m}^{2}$. However, most of these data were acquired for a 
limited fluence range and / or elevated surface temperatures. More recent analysis of the ion beam irradiation data at IPP Garching [9-11] gives an insight in the fuel retention mechanisms in beryllium. The thermal desorption spectrometry (TDS) data exhibit two high-energy peaks at $\mathrm{T}_{\mathrm{TDS}}$ of 770 and $840 \mathrm{~K}$, attributed to pre-existing and ion-induced trap sites, which saturate at a fluence of $\sim 10^{21} \mathrm{D}^{+} / \mathrm{m}^{2}$, consistent with [8]. At a higher fluence, additional low-energy peaks at $\mathrm{T}_{\mathrm{TDS}}$ of 440 and $470 \mathrm{~K}$ appear, leading to the further uptake of the $\mathrm{D}$ inventory. These D trapping sites are characterized as so-called "supersaturation" binding states, referring to the already saturated high-energy states. For room temperature (RT) exposures and at fluences higher than $\sim 10^{22} \mathrm{D}^{+} / \mathrm{m}^{2}$ the supersaturation states dominate the overall hydrogen inventory in bulk beryllium [12]. At $\mathrm{T}_{\mathrm{TDS}}$ of $500-600 \mathrm{~K}$ a further peak is observed. This peak is attributed to the decomposition of beryllium deuteride, $\mathrm{BeD}_{2}$, which is formed either during the sample exposure or during the TDS temperature ramp through the transition from the supersaturation states $[9,10,13]$. The existence of the low-energy trapping sites can explain the scatter of data for similarly high fluences but different exposure temperatures in [8].

The Be surface morphology undergoes significant changes at high fluences, such as roughening, appearance of nano-sized bubbles presumably filled with deuterium and, at even higher fluences, interconnection of bubbles and further erosion leading to the formation of an open pore network on a micrometer scale $[8,14,15]$.

Be:H co-deposits are formed from beryllium eroded under the plasma bombardment. If ITER will forgo the deployment of carbon-based plasma-facing materials, the co-deposition with beryllium is believed to be the dominant fuel retention mechanism [16]. Varying the Be deposition flux, the incident ion energy and the substrate temperature, a scaling for the $\mathrm{D} / \mathrm{Be}$ ratio in co-deposited layers was obtained in PISCES-B $[17,18]$.

A radiative cooling of the divertor by the impurity seeding will be necessary in ITER to keep the particle and power loads to the target at an acceptable level [1]. Nitrogen and argon are the main candidates for this role. The influence of nitrogen on the erosion, formation of co- 
deposited layers and fuel retention of beryllium was recently studied in PISCES-B [19, 20]. A significant storage of nitrogen was observed in the plasma chamber, which subsequently could be recovered by the operation in deuterium but not in helium. This underlines the chemical activity of nitrogen and calls for its cautious use. The beryllium sputtering was somewhat reduced, presumably due to the formation of a protective Be:N surface layer. While the layers on the target surface remained electrically conductive, the thick Be:N:D codeposited layers on a collector probe were insulating. This may cause arcing on the deposition dominated surfaces in ITER. There was no significant influence of nitrogen on the fuel retention in co-deposited layers.

This contribution is aimed on the assessment of the influence of argon as plasma impurity on the PMI properties of beryllium. The Be-compatible PISCES-B linear plasma device was once again used to conduct these studies. Previous investigations on this topic in PISCES-B [21] indicated a significant influence of argon on the properties of co-deposited layers. In [21], the Be seeding was used to enhance the deposition rates on the collector sample. In the studies presented here, the erosion from the Be target in pure and argon seeded deuterium plasmas, the subsequent re-deposition on the collector sample and the deuterium retention in both the Be target and the deposited layer were investigated in a self-consistent manner. No Be seeding was used, so that only Be sputtered from the target was deposited on the collector sample.

The second aim of this study was investigating aluminium as a proxy for toxic beryllium for the relevant PMI studied in devices non-compatible with beryllium. Many of the physical and chemical properties of these elements are similar [22], suggesting aluminium as a potential substitute for beryllium.

\section{Experimental procedure and post-mortem analyses}

The PISCES-B linear plasma device is installed inside an air-tight enclosure and is therefore capable of PMI studies with toxic beryllium [23]. Figure 1 illustrates the experimental set-up 
on PISCES-B. Targets of press-sintered beryllium (Brush Wellman S-65C) were annealed at $920 \mathrm{~K}$ for $20 \mathrm{~min}$ and then exposed to deuterium plasma discharges with a fraction of argon ions of zero (reference case), 3\% and 10\%. The fraction of argon ions was monitored spectroscopically as described in [24]. Experiments with the Al target were only performed in pure D plasma. The material eroded from the target was collected on the tungsten substrate installed in the witness probe (WP) manipulator (figure 1). The WP sample is shielded from the direct cross-field transport from the plasma column. The plasma parameters for different exposures are given in table 1. Other exposure parameters were reproducible: an incident ion energy at the biased target of $100 \mathrm{eV}$, target and WP sample temperatures of $320 \pm 15$ and $295 \pm 5 \mathrm{~K}$, respectively, and an incident ion flux of $4 \times 10^{22} \mathrm{D}^{+} / \mathrm{m}^{2} \mathrm{~s}$. The collected fluence was about $1 \times 10^{26} \mathrm{D}^{+} / \mathrm{m}^{2}$, corresponding to about one hour of exposure, except for one case with pure deuterium plasma, where the fluence was doubled to collect more material on the witness sample.

The erosion from the target was monitored in-situ by optical spectroscopy and integrally by the mass-loss method. The surface morphology after exposure was investigated by scanning electron microscopy (SEM). The deuterium retention in the target and in the layer deposited on the WP sample was studied by ex-situ TDS with a constant heating ramp of $0.5 \mathrm{~K} / \mathrm{s}$ up to $930 \mathrm{~K}$ for beryllium and $770 \mathrm{~K}$ for aluminium. The total amount of retention was determined from integrated desorption spectra at mass $4\left(\mathrm{D}_{2}\right)$ and $3(\mathrm{HD})$. The deuterium retention in other molecules was at most of a few additional percent and was therefore neglected. Postmortem nuclear reaction analysis (NRA) was applied to determine the amount of material deposited on the witness sample using the following reactions: ${ }^{3} \mathrm{He}(\mathrm{D}, \mathrm{p}){ }^{4} \mathrm{He}$ for deuterium and ${ }^{3} \mathrm{He}(\mathrm{Be}, \mathrm{p}){ }^{11} \mathrm{~B}$ for beryllium, both using a $2 \mathrm{MeV}{ }^{3} \mathrm{He}$ beam, and ${ }^{27} \mathrm{Al}(\mathrm{D}, \mathrm{p}){ }^{28} \mathrm{Al}$ for aluminium with a $1.4 \mathrm{MeV} \mathrm{D}$ beam. The NRA technique was applicable to determine the amounts of Be and D deposited on the witness sample in the case of mixed D/Ar plasmas, while in the case of pure D plasma the amount of deposition was below the detection limit of 
NRA. Therefore, in the latter case, the Be amount was estimated using the results of the massloss method, assuming that the amount of Be deposited on the witness sample scales linearly with the amount of Be eroded from the target. This assumption should be valid for the reproducible exposure conditions.

Argon as noble gas did not show any long-term effects on the experimental conditions. With the argon gas inlet shut off, its fraction in plasma quickly decayed to a level below $\sim 1 \%$ detectable by the spectroscopy system. The amount of argon stored in the target samples and in the co-deposited layers was below the detection limit of the TDS system of $\sim 10^{19} \mathrm{~m}^{-2}$.

\section{Results}

Evaluated values of the target sputtering yield, retention in the material bulk and in the codeposited layers for the entire experimental series are summarized in table 1 . The following subsections will describe the observed phenomena in detail.

\subsection{Surface morphology}

SEM images of the target for different plasma compositions are shown in figure 2. For the case of pure deuterium plasma the surface exhibits a typical for PISCES-B [5, 25] fine-scale grass-like structure with a diameter of tendrils of $10-100 \mathrm{~nm}$. The admixture of 3\% Ar leads to the formation of a cone-like structure with a typical size of $\sim 1 \mu \mathrm{m} .10 \%$ Ar result in a structure with a typical scale of several $\mu \mathrm{m}$ and reduced roughness. Taken from a different experimental series, figure 2(e) illustrates that the beryllium surface exposed to pure argon plasma remains relatively smooth. In general, a gradual smoothing out of the surface with the increasing fraction of argon was observed.

The amount of material re-deposited on the WP sample corresponds to a layer thickness of $\sim 10 \mathrm{~nm}$, which is below the resolution capability of the used SEM system.

\subsection{Deuterium retention in beryllium targets}

TDS spectra for beryllium targets exposed to plasmas with different fractions of argon are shown in figure 3. Experiments with pure deuterium plasma were performed twice with 
different fluences of $1.2 \times 10^{26} \mathrm{D}^{+} / \mathrm{m}^{2}$ and $2.3 \times 10^{26} \mathrm{D}^{+} / \mathrm{m}^{2}$. The corresponding TDS spectra exhibit typical features [9-12] with the high-energy pre-existing or ion-induced trapping sites at $\mathrm{T}_{\mathrm{TDS}}$ of $700-800 \mathrm{~K}$, the low-energy supersaturation binding states at $\mathrm{T}_{\mathrm{TDS}}$ of $480-580 \mathrm{~K}$ and, in-between at $\mathrm{T}_{\mathrm{TDS}}$ of $570-670 \mathrm{~K}$, a signature of the $\mathrm{BeD}_{2}$ decomposition. Note that the release temperature of the supersaturation binding states is here by $50-100 \mathrm{~K}$ higher than typically observed in ion beam experiments (section 1).

Though investigating the fluence dependence was not the primary purpose of this study, it is worth mentioning that the total $\mathrm{D}$ retention increases for the two data points of pure $\mathrm{D}$ plasma (table 1). This increase is solely due to a higher inventory in the low-energy supersaturation binding states. The enhanced surface roughness and, consequently, the larger effective surface area can be the reason for the higher retention.

Adding 3\% Ar to D plasma leads to a reduction in the total D retention of about $20 \%$ (table 1 ). A shoulder on the right-hand side of the supersaturation TDS peak at about $570 \mathrm{~K}$ becomes visible, indicating two low-energy binding states in agreement with [10]. An increase of the Ar fraction to $10 \%$ results in a drop of D retention of more than $50 \%$. The peak at $570 \mathrm{~K}$ is of a higher amplitude than the peak at $500 \mathrm{~K}$. This appearance of the supersaturation doublepeak is similar to ones from beryllium samples irradiated by ion beams at a fluence of (12) $\times 10^{21} \mathrm{D}^{+} / \mathrm{m}^{2}[10,15]$. This fluence corresponds to the marginal saturation of the highenergy trapping sites and the initial appearance of the low-energy supersaturation binding states.

\subsection{Erosion of beryllium targets}

Figures $4 \mathrm{a}$ ) and $4 \mathrm{~b}$ ) show the intensity of the Be I line at $457.3 \mathrm{~nm}$ in front of the target along the plasma column for the cases of pure deuterium and deuterium mixed with $10 \%$ argon plasmas, respectively. The signal is normalized to the $D_{\gamma} 433.9 \mathrm{~nm}$ line intensity to compensate for the inhomogeneous distribution of the electron density along the plasma column and can be understood as a relative measure for the beryllium erosion from the target. 
The intensity drops in the course of the experiment with pure deuterium plasma, indicating a reduction of the beryllium erosion. In contrast, it remains at the same level when argon is admixed. The Be I line intensity decays when moving away from the target. This decay is due to the ionization of sputtered $\mathrm{Be}$ atoms and their losses from the plasma column. The characteristic length of the decay increases when the experiment with pure D plasma proceeds. This apparent increase of the Be penetration can be explained by a geometrical effect of the surface with increased roughness, as seen in the SEM image (figure 2a). The increased surface roughness results in a preferential erosion of $\mathrm{Be}$ atoms with a higher component of the velocity parallel to the magnetic field, while particles with a higher perpendicular velocity are predominantly trapped in the surface protrusions ([25] and figure 5 in [6]). On the contrary, in the case with $10 \%$ Ar the decay length does not significantly change, in agreement with the SEM observations of the target surface remaining relatively smooth (figures $2 \mathrm{c}$ and $2 \mathrm{~d}$ ).

The Be sputtering yield values determined by the mass loss technique are given in table 1 . The values are significantly lower than the sputtering yields predicted by the TRIM code, which is typical for the experiments in PISCES-B (section 1). E.g. for the sample presented in figures $2 \mathrm{a}$ and $4 \mathrm{a}$ it is a factor of 22 lower. Other sample exposed to pure D plasma has a sputtering yield a factor of 64 lower than the TRIM value. This sample was exposed to denser plasma, which probably increased the fraction of Be re-deposited on the target, effectively reducing the sputtering yield.

Note that the TRIM sputtering yield of beryllium at an incident ion energy of argon ions of $100 \mathrm{eV}$ is somewhat lower than that for deuterium ions: 0.018 and 0.031 , respectively [3]. Therefore, adding a few percent argon to the deuterium ions should not significantly change the effective sputtering yield. However, in the experiments we observed a substantial increase of the Be sputtering when Ar was added, with yields significantly closer to the TRIM values. 
The measured Be sputtering yield for pure argon plasma is in good agreement with the TRIM prediction [25].

\subsection{Formation and fuel retention of beryllium co-deposited layer}

A fraction of material sputtered from the target is subsequently deposited on the witness probe sample made of tungsten and kept at room temperature (figure 1). Evidently, the higher erosion from the target in experiments with argon results in the larger amount of deposited material. The appearance of the TDS spectra of the co-deposited layers is, however, similar in the cases with and without argon (figure 5). The spectra exhibit similar features as observed e.g. in $[21,26]$ with a low-temperature peak, presumably corresponding to the supersaturation binding states, and a shoulder towards higher temperatures corresponding to the decomposition of $\mathrm{BeD}_{2}$. The total amount of co-deposited deuterium is comparable for the cases with and without argon, which results in incrementally lower $\mathrm{D} / \mathrm{Be}$ values in the experiments with higher argon fractions in the plasma (table 1).

\subsection{Comparison of aluminium as beryllium}

An aluminium target was exposed to pure deuterium plasma at conditions similar to the experiments with the beryllium targets. Aluminium exhibited several phenomena similar to beryllium: the Al surface also developed a fine-scale grass-like structure (figure 2f); the measured Al sputtering yield was a factor of 28 lower than the TRIM prediction (table 1); the spectroscopy of Al I line at $396.1 \mathrm{~nm}$ (figure 4c) indicates a drop of Al sputtering during the exposure and an apparent increase of the penetration length of $\mathrm{Al}$ into the plasma column. However, the TDS characteristics of the Al target indicate different D retention mechanisms than in Be (figure 3a). The TDS spectra of the co-deposited layers with Al and Be show, in contrast, certain similarities both in the shape and in the amplitude (figure 5).

\section{Summary and discussion}

Sputtering yields of beryllium when exposed to pure deuterium plasma in PISCES-B are persistently lower than the values predicted by the TRIM code. As described in section 1, this 
deviation is attributed to (i) the evolution of the surface morphology with a significantly enhanced surface roughness which increases the fraction of promptly re-deposited particles and (ii) the protective effect of deuterium stored in the surface layer. The admixture of argon to the plasma has an impact on both of these effects. Firstly, the surface remains relatively smooth and, secondly, the amount of deuterium stored in the surface is significantly reduced. Both circumstances result in an enhancement of the Be sputtering comparing to the case of pure D plasma. Deuterium remaining in the surface can account for the sputtering yield still somewhat lower than the TRIM prediction. A fraction of sputtered Be is redeposited on the target, which effectively reduces the sputtering yield determined by the mass-loss method. This can also add to the remaining discrepancy between the measured and the predicted yields.

The admixture of Ar to the plasma significantly reduces the D retention in the Be target, mainly due to a lower inventory in the supersaturation binding states. One can argue that the D retention scales with the effective surface area, which is reduced in the presence of Ar due to a lower surface roughness. However, the different structure of the low-energy TDS peaks indicates significant changes in the retention mechanism in the presence of Ar. A similar effect of the D inventory reduction by argon was observed for mixed carbon-beryllium layers and was explained by the ion-induced desorption [27].

A higher Be sputtering yield in the presence of Ar results in a higher growth rate of deposited layers. However, at least for the conditions of PISCES-B, it does not result in a significant overall uptake of $\mathrm{D}$ in the layers due to lower D/Be values. The scaling for PISCES-B [16] predicts $\mathrm{D} / \mathrm{Be} \propto(\mathrm{Be} \text { deposition rate })^{-0.59}$. However, this power law is not strong enough to explain the D retention remaining at a similar level without or with argon. It suggests that the co-deposited layers are directly affected by the bombardment by Ar, i.e. through the ioninduced desorption of $\mathrm{D}$. 
The low-energy binding states may be of concern for the ITER main wall, for which a bake temperature of $510 \mathrm{~K}$ is foreseen [28]. This temperature may be marginal for an effective desorption of implanted and co-deposited deuterium. E.g. for the outgassing of the Be codeposition samples in figure 5 , about $30 \%$ of deuterium remains trapped at $510 \mathrm{~K}$, while at $620 \mathrm{~K}$, the bake temperature of the divertor, more than $90 \%$ is desorbed.

In summary, the admixture of a few percent argon to deuterium plasma causes some significant effects on the PMI properties of beryllium. However, the implications for ITER are expected to be rather moderate.

Aluminium exhibits similar phenomena to beryllium with respect to the evolution of the surface morphology and the reduction of sputtering when exposed to deuterium plasma. The D retention mechanisms, however, appear to be different. With these restrictions, Al can potentially be employed as a Be substitute in the experiments incapable of handling toxic Be. This would also require a further refinement of the measurement techniques such as optical spectroscopy and ion beam analysis, since there is significantly less experience in the application of those techniques for $\mathrm{Al}$ than for Be.

\section{Acknowledgements}

The authors would like to thank the engineering staff of the PISCES lab at UCSD for their excellent support in the preparation and the execution of the experiments. This work was supported by the EU-US bilateral collaboration on mixed-material plasma-surface interaction effects for ITER.

\section{References}

[1] Progress in the ITER Physics Basis 2007 Nucl. Fusion 47 S1

[2] Federici G et al 2012 Comprehensive Nuclear Materials, ed R Konings (Elsevier Science) p 621

[3] Eckstein W 2002 Calculated sputtering, reflection and range values Max-Planck-Institut für Plasmaphysik Report IPP 9/132 (Garching) 
[4] Doerner R P et al 1998 J. Nucl. Mater. 25751

[5] Doerner R P et al 2013 J. Nucl. Mater. http://dx.doi.org/10.1016/j.jnucmat.2013.01.045

[6] Kreter A et al 2008 Plasma Phys. Control. Fusion 50095008

[7] Kreter A et al 2006 Plasma Phys. Control. Fusion 481401

[8] Anderl R A et al 1999 J. Nucl. Mater. 2731

[9] Reinelt M and Linsmeier Ch 2009 J. Nucl. Mater. 390-391 568

[10] Reinelt M et al 2009 New Journal of Physics 11043023

[11] Oberkofler M and Linsmeier Ch 2011 J. Nucl. Mater. 415 S724

[12] Haasz A A and Davis J W 1997 J. Nucl. Mater. 241-243 1076

[13] Doerner R P et al 2009 J. Nucl. Mater. 390-391 681

[14] Anderl R A et al 1992 J. Nucl. Mater. 196-198 986

[15] Markin A V et al 1996 J. Nucl. Mater. 233-237 865

[16] Roth J et al 2008 Plasma Phys. Control. Fusion 50103001

[17] De Temmerman G et al 2008 Nucl. Fusion 48075008

[18] De Temmerman G and Doerner R P 2009 Nucl. Fusion 49042002

[19] Dittmar T et al 2011 Phys. Scr. T145 014009

[20] Dittmar T et al 2013 J. Nucl. Mater. http://dx.doi.org/10.1016/j.jnucmat.2013.01.215

[21] De Temmerman G et al 2009 J. Nucl. Mater. 390-391 564

[22] Marot L et al 2013 Fusion Eng. Des. http://dx.doi.org/10.1016/j.fusengdes.2013.04.040

[23] Doerner R P et al 2004 Phys. Scr. T111 75

[24] Kreter A et al 2011 J. Nucl. Mater. 417651

[25] Doerner R P et al these proceedings

[26] Sugiyama K et al 2011 J. Nucl. Mater. 415 S731

[27] Kreter A et al 2009 Phys. Scr. T138 014012

[28] Pitts R A et al 2009 Phys. Scr. T138 014001 
Table 1. Summary of the experiments performed in PISCES-B within this study. The first column indicates the plasma composition and the target material. Electron density and temperature are given in columns 2 and 3, respectively. The total incident ion fluence collected on the target (column 4) was somewhat higher than that corresponding to the exposure of the witness probe (WP) sample (column 5), as the WP manipulator was introduced about $800 \mathrm{~s}$ after the start of the target exposure. The sputtering yield determined by the mass loss technique (column 6) is compared with the value predicted by the TRIM code [3] for the applied conditions (column 7). The total D retention in the target (column 8) and in the co-deposited layer on the WP sample (column 9) are from the integration of mass 4 $\left(\mathrm{D}_{2}\right)$ and mass $3(\mathrm{HD})$ signals of the TDS spectrum. The D/Be ratios for the co-deposited layers on the collector samples (column 10) were estimated combining the TDS, NRA and mass loss techniques, as not all of the techniques were applicable to all samples.

\begin{tabular}{|c|c|c|c|c|c|c|c|c|c|}
\hline Experiment & $\begin{array}{l}\text { Electron } \\
\text { density } \\
{\left[\mathrm{m}^{-3}\right]}\end{array}$ & $\begin{array}{l}\text { Electron } \\
\text { temperatur } \\
\mathrm{e}[\mathrm{eV}]\end{array}$ & $\begin{array}{l}\text { Ion } \\
\text { fluence } \\
\text { to target } \\
\text { total } \\
{\left[\mathrm{m}^{-2}\right]}\end{array}$ & $\begin{array}{l}\text { Ion fluence } \\
\text { to target } \\
\text { during WP } \\
\text { sample } \\
\text { exposure } \\
{\left[\mathrm{m}^{-2}\right]}\end{array}$ & $\begin{array}{l}\text { Sputtering } \\
\text { yield from } \\
\text { mass loss }\end{array}$ & $\begin{array}{l}\text { Ratio of } \\
\text { sputtering } \\
\text { yields } \\
\text { TRIM to } \\
\text { experiment }\end{array}$ & $\begin{array}{l}\mathrm{D} \\
\text { retention } \\
\text { in target } \\
{\left[\mathrm{m}^{-2}\right]}\end{array}$ & $\begin{array}{l}\mathrm{D} \\
\text { retention } \\
\text { on WP } \\
\text { sample } \\
{\left[\mathrm{m}^{-2}\right]}\end{array}$ & $\begin{array}{l}\mathrm{D} / \mathrm{Be} \\
\text { on WP } \\
\text { sample }\end{array}$ \\
\hline $\mathrm{D} \rightarrow \mathrm{Be}$ & $5.6 \cdot 10^{18}$ & 3 & $1.2 \cdot 10^{26}$ & $1.0 \cdot 10^{26}$ & $0.5 \cdot 10^{-3}$ & 64 & $3.2 \cdot 10^{21}$ & N/A & N/A \\
\hline $\begin{array}{l}\mathrm{D} \rightarrow \mathrm{Be} \\
\text { (double fluence) }\end{array}$ & $4.0 \cdot 10^{18}$ & 5 & $2.3 \cdot 10^{26}$ & $2.0 \cdot 10^{26}$ & $1.4 \cdot 10^{-3}$ & 22 & $4.5 \cdot 10^{21}$ & $3.6 \cdot 10^{20}$ & $\approx 0.5$ \\
\hline $\mathrm{D}+0.03 \mathrm{Ar} \rightarrow \mathrm{Be}$ & $4.0 \cdot 10^{18}$ & 5 & $1.2 \cdot 10^{26}$ & $1.0 \cdot 10^{26}$ & $3.1 \cdot 10^{-3}$ & 9.9 & $2.8 \cdot 10^{21}$ & $0.7 \cdot 10^{20}$ & $\approx 0.2$ \\
\hline $\mathrm{D}+0.1 \mathrm{Ar} \rightarrow \mathrm{Be}$ & $4.0 \cdot 10^{18}$ & 5 & $1.2 \cdot 10^{26}$ & $1.0 \cdot 10^{26}$ & $9.2 \cdot 10^{-3}$ & 3.4 & $1.5 \cdot 10^{21}$ & $1.3 \cdot 10^{20}$ & $\approx 0.1$ \\
\hline $\mathrm{D} \rightarrow \mathrm{Al}$ & $5.6 \cdot 10^{18}$ & 3 & $1.2 \cdot 10^{26}$ & $1.0 \cdot 10^{26}$ & $2.8 \cdot 10^{-4}$ & 28 & $1.9 \cdot 10^{21}$ & $1.4 \cdot 10^{20}$ & N/A \\
\hline
\end{tabular}




\section{Biased Be target}

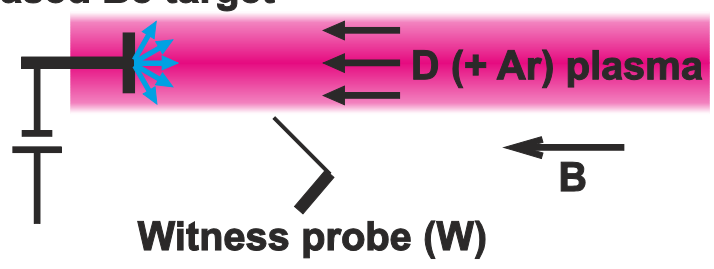

Figure 1. Schematic view of the experimental set-up in PISCES-B used in this study. 

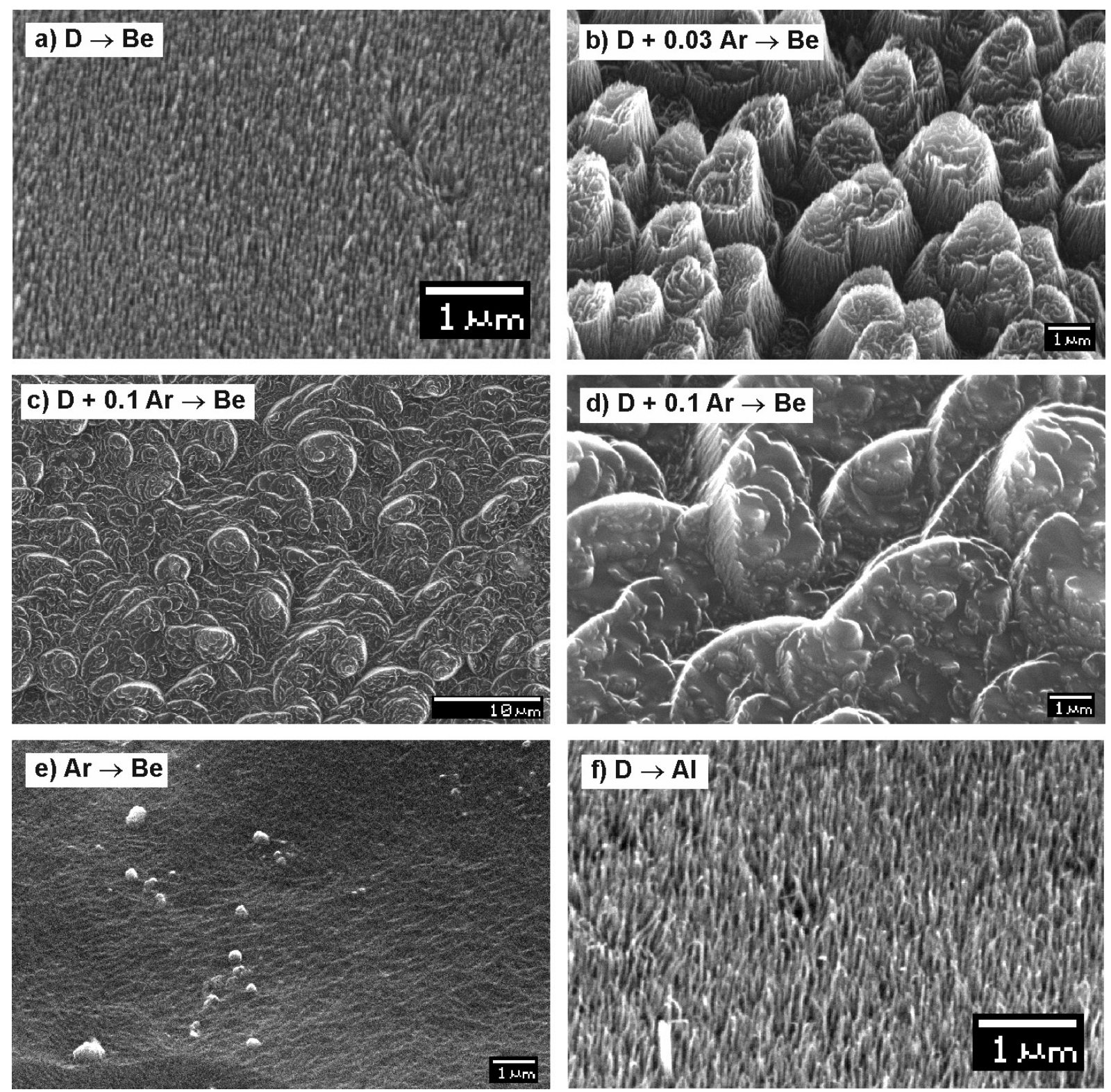

Figure 2. SEM images of (a) a beryllium target exposed to pure deuterium plasma, (b) Be exposed to D plasma with $3 \% \mathrm{Ar}$, (c) and (d) Be exposed to D plasma with $10 \%$ Ar with two different magnifications, (e) Be exposed to pure Ar plasma and (f) Al exposed to pure D plasma. 

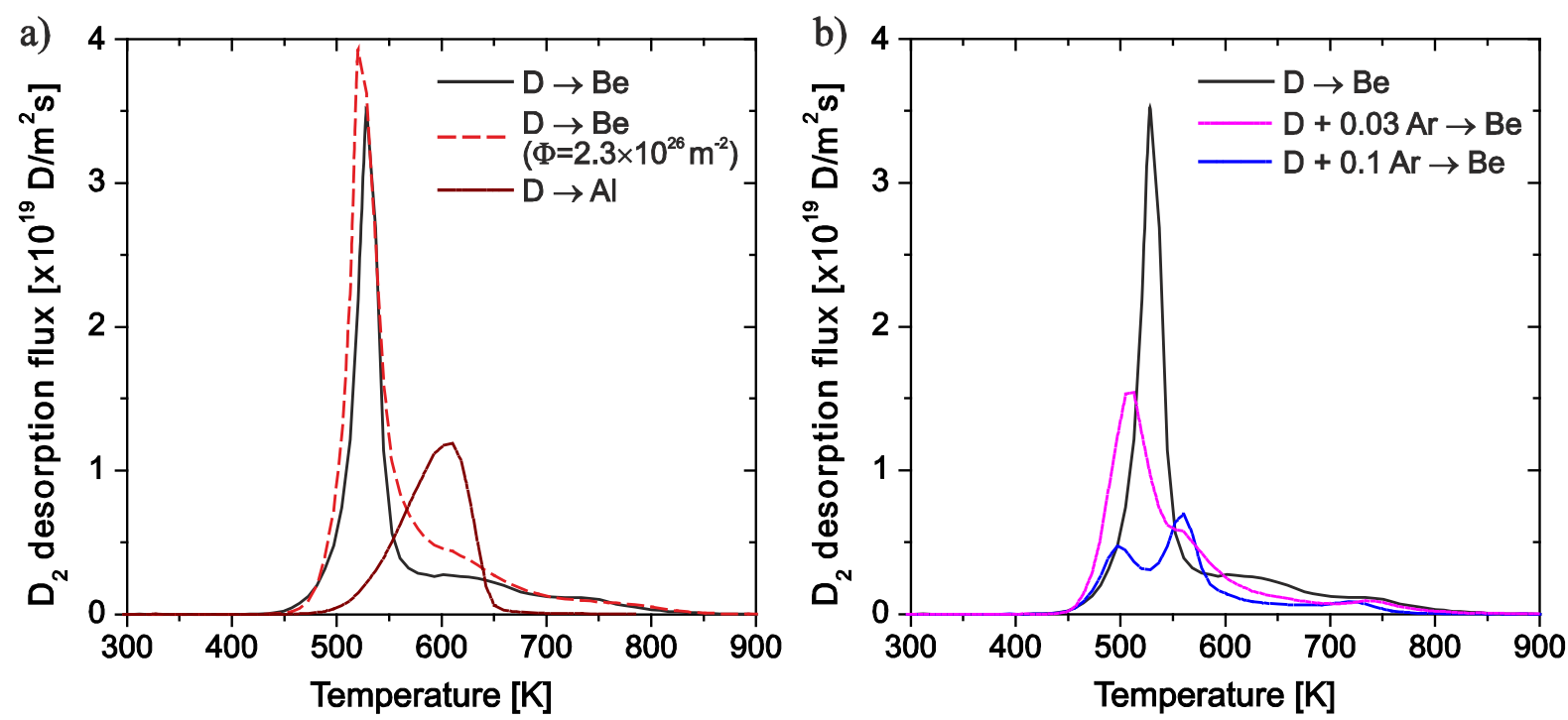

Figure 3. TDS spectra of mass $4\left(\mathrm{D}_{2}\right)$ from target samples exposed to (a) pure deuterium plasma and (b) plasma with different fractions of argon. The legend shows the plasma composition and the sample material. The total incident ion fluence was $1.2 \times 10^{26} \mathrm{~m}^{-2}$ except for one case with $2.3 \times 10^{26} \mathrm{~m}^{-2}$ as indicated in the legend. The TDS heating ramp rate was 0.5 $\mathrm{K} / \mathrm{s}$. 

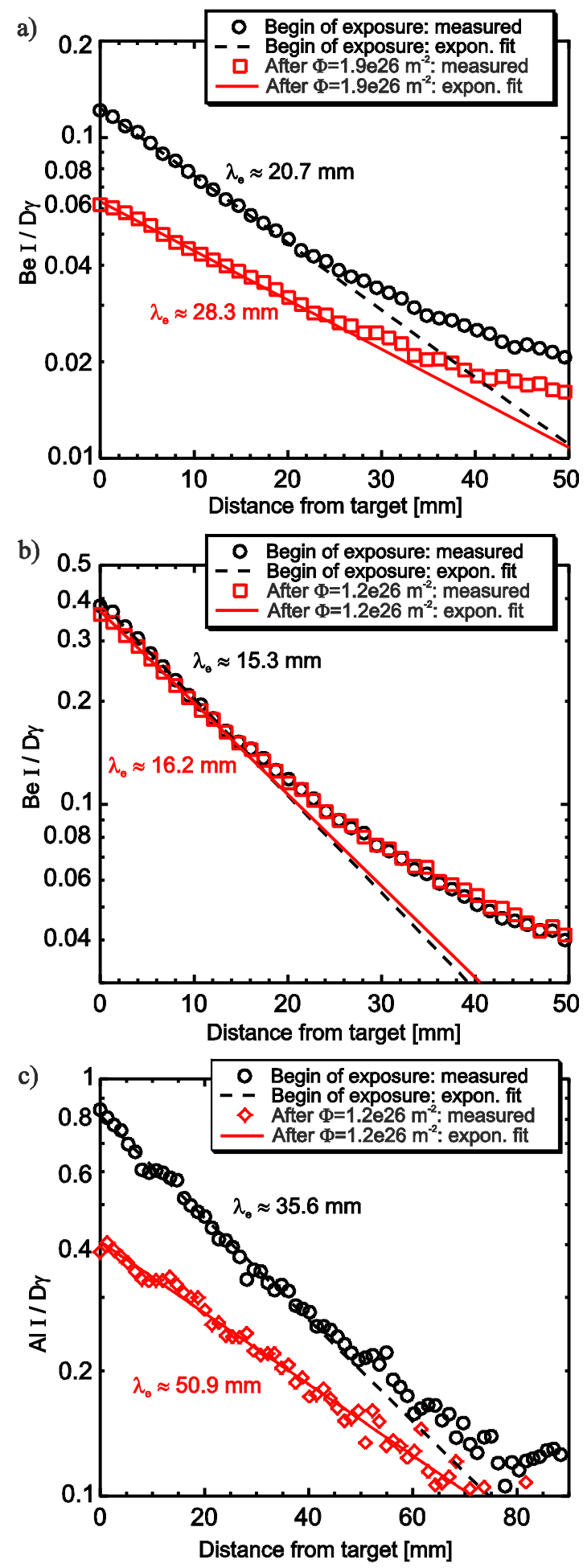

Figure 4. Intensity distribution in front of the target along the plasma column of Be I line at $457.3 \mathrm{~nm}$ (a) for pure D plasma and (b) for 10\% Ar added to D plasma and (c) Al I line at $396.1 \mathrm{~nm}$ for pure $\mathrm{D}$ plasma. The signals are normalized to the $\mathrm{D}_{\gamma} 433.9 \mathrm{~nm}$ line intensity. Data are given for the beginning and close to the end of the exposure. Measured distributions 
(symbols) were fitted by decaying exponential functions (straight lines). The resulting characteristic lengths $\lambda_{\mathrm{e}}$ are indicated. 


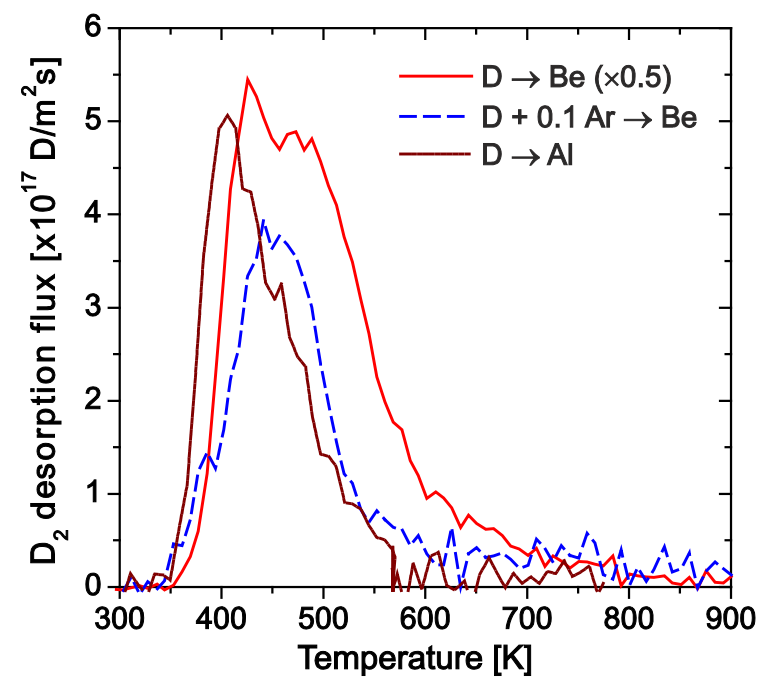

Figure 5. TDS spectra of mass $4\left(\mathrm{D}_{2}\right)$ from co-deposited layers on the WP samples. The legend shows the plasma composition and the target sample material. The signal from the experiment with Be exposed to pure $\mathrm{D}$ was multiplied by a factor 0.5 to compensate for the twice as high fluence as compared with two other curves. The TDS heating ramp rate was 0.5 $\mathrm{K} / \mathrm{s}$. 